\title{
Microscopic quantum generalization of classical Liénard oscillators
}

\author{
Srijan Bhattacharyya, ${ }^{1}$ Arnab Ghosh,, , ศ and Deb Shankar Ray ${ }^{2}$ \\ ${ }^{1}$ Indian Institute of Technology Kanpur, Kanpur,Uttar Pradesh 208016, India \\ ${ }^{2}$ Indian Association for the Cultivation of Science, Jadavpur, Kolkata 700032, India
}

\begin{abstract}
Based on a system-reservoir model and an appropriate choice of nonlinear coupling, we have explored the microscopic quantum generalization of classical Liénard systems. Making use of oscillator coherent states and canonical thermal distributions of the associated c-numbers, we have derived the quantum Langevin equation of the reduced system which admits of single or multiple limit cycles. It has been shown that detailed balance in the form of fluctuation-dissipation relation preserves the dynamical stability of the attractors even in case of vacuum excitation. The quantum versions of Rayleigh, Van der Pol and several other variants of Liénard oscillators are derived as special cases in our theoretical scheme within a mean-field description.
\end{abstract}

\section{INTRODUCTION}

Dissipation is an intriguing issue in physical sciences [1. While its incorporation in dynamical problems from the classical point of view is largely phenomenological, the approach turns out to be untenable because of its violation of uncertainty principle, when the system is quantized. The problem is circumvented by coupling the system to a reservoir with infinite degrees of freedom kept at a finite temperature, which allows the fluctuations of the reservoir to act on the system inducing its dissipation or decay [2. To maintain thermal equilibrium of the system in contact with reservoir, fluctuation and dissipation get connected through fluctuation-dissipation theorem [3]. The system-reservoir model describing a dissipative quantum system lies at the heart of the problems related to macroscopic quantum coherence [4, quantized tunneling in condensed matter physics [5], relaxation processes in quantum optics [6] and magnetic resonance spectroscopy [7, to name a few. An important note in this context is that the dissipative term appearing in the reduced stochastic equation of motion for the system is by and large linear, arising out of linear coupling between the system and the reservoir [8-10]. Introduction of nonlinearity in the dissipative term in a classical system without any noise, on the other hand, may lead to a complete modification of the dynamics. Such nonlinearity in dissipation may result in a force which acts on the system as an intrinsic source of creation of limit cycle [11, an asymptotically isolated trajectory in phase space. Liénard equation represents a prototypical paradigm for such a classical nonlinear dissipative system of which two special cases are Van der Pol [12] and Rayleigh oscillators [13]. The object of the present paper is the search for a quantum analog of such classical Liénard oscillator within the framework of systemreservoir theory.

Liénard system is widely used in describing many oscillating circuits in the development of radio and vacuum tube technology [11-13]. The second order differential

\footnotetext{
* arnab@iitk.ac.in
}

equation describing classical Liénard system is

$$
\ddot{x}+f(x) \dot{x}+g(x)=0 .
$$

If $f(x)$ and $g(x)$ follow the Liénard's theorem, then the system has at least one unique, stable limit cycle around its origin in the phase plane [11]. The theorem assures that the odd function $g(x)$ acts as a restoring force that tries to reduce any displacement. While assumptions on the even function $f(x)$ indicate that it acts as a negative damping at small displacement and positive damping on large displacement. As a result, small oscillations are forced up while large ones are damped down. Thus, it is not difficult to anticipate that the system will settle into a self-sustained oscillation of some intermediate amplitude. Apart from mono-rhythmic models with van der Pol or Rayleigh oscillators, Liénard equation depending on the form of the polynomial $f(x)$, may admit bi-rhythmic solutions. The equation has been generalized further to Liénard-Smith-Levinson oscillator form that includes the multiple limit cycles [14 16].

The aim of our present work is to propose a microscopic quantum description of the classical Liénard oscillator in the system-reservoir model and subsequent generalization to its several variants. To capture the nature of Liénard system, we have defined the interaction Hamiltonian containing appropriate nonlinear coupling terms. The time evolution of the dynamics is followed by Heisenberg equation of motion to obtain operator Langevin equation for the reduced system. The noise and the nonlinear dissipation originating from the systemreservoir coupling are shown to follow the fluctuationdissipation relation. Our approach is based on a cnumber Langevin equation using harmonic oscillator coherent states and canonical thermal distribution of the associated c-numbers for the reservoirs. This ensures that the overall system is thermodynamically closed. The c-number Langevin equation plays a key role in describing the noisy quantum Liénard system. Van der Pol and Rayleigh oscillators are also depicted with a quantum noise term as model examples. Finally our proposal is generalized for several model cases of Eq. (1).

The present work is organized as follows: In Sec. II we introduce the model Hamiltonian to derive reduced 
dynamics for the system in terms of operator Langevin equation. In the next section oscillator coherent states are used to construct quantum Langevin equation with c-number noise. Microscopic realization of classical Van der Pol and Rayleigh oscillator are discussed in Sec. IV. Later on further generalization of the theoretical scheme for arbitrary form of classical Liénard systems are presented. Finally we conclude in Sec. V.

\section{QUANTUM ANALOG OF CLASSICAL LIÉNARD OSCILLATOR}

Our search for microscopic description of classical Liénard oscillation is based on quantum harmonic oscillator, whose Hilbert space is given by Fock states $|n\rangle$, where $n$ is the number of quanta in that state. The form of the total system-reservoir Hamiltonian is given by

$\hat{H}=\hbar \omega_{0} \hat{a}^{\dagger} \hat{a}+\sum_{k} \hbar \omega_{k} \hat{n}_{k}+i \hbar \sum_{k} g_{k}\left[\left(\hat{a}^{\dagger}\right)^{n+1} \hat{b}_{k}-\hat{a}^{n+1} \hat{b}_{k}^{\dagger}\right]$,

where the first term is the unperturbed system Hamiltonian $\hat{H}_{S}=\hbar \omega_{0} \hat{a}^{\dagger} \hat{a}$, the second term represents the free reservoir (bath) Hamiltonian $\hat{H}_{R}=\sum_{j} \hbar \omega_{j} \hat{b}_{j}^{\dagger} \hat{b}_{j}$, consisting of large number of harmonic oscillators, and the last term describes the interaction Hamiltonian for $n \in \mathbb{Z}^{+}$. Here $\hat{a}$ and $\hat{b}_{k}$ are the annihilation operators for system and reservoir respectively which fulfil the following commutation relations:

$$
\left[\hat{a}, \hat{a}^{\dagger}\right]=1, \quad\left[\hat{b}_{m}, \hat{b}_{n}^{\dagger}\right]=\delta_{m n}, \quad\left[\hat{a}, \hat{b}_{k}\right]=0
$$

The elementary exchange of energy between the system and the reservoir consists of single quantum absorption from the $k$-th bath mode and simultaneous creation of $(n+1)$ quanta of excitation in the system mode and vice-versa.

Since our object here is to recover the dissipative dynamics of the Liénard oscillation from the systemreservoir Hamiltonain Eq. (2) for which dissipation is always accompanied by an internal quantum noise, we expect a modification for Eq. (1) in the following form in a c-number description;

$$
\ddot{x}+f(x) \dot{x}+\omega_{0}^{2} x=\eta(t) .
$$

Here $\eta(t)$ is a Gaussian white $\delta$-correlated quantum noise with zero mean. In other words, we look for a connection between the nonlinear dissipation $f(x) \dot{x}$ and stochastic noise term $\eta(t)$ which allows the dynamical system to admit stable but noisy limit cycle oscillations.

From Eq. (2), using the commutation relations [Eq. (3)], we can easily evaluate the Heisenberg operator equations for the system and bath degrees of freedom as

$$
\dot{\hat{a}}(t)=-i \omega_{0} \hat{a}(t)+(n+1) \sum_{k} g_{k}\left(\hat{a}^{\dagger}\right)^{n} \hat{b}_{k}(t),
$$

and

$$
\dot{\hat{b}}_{j}(t)=-i \omega_{j} \hat{b}_{j}(t)-g_{j} \hat{a}^{n+1}(t)
$$

respectively. Formally integrating Eq. (6), we get

$$
\hat{b}_{j}(t)=\hat{b}_{j} e^{-i \omega_{j} t}-g_{j} \int_{0}^{t} \hat{a}^{n+1}\left(t^{\prime}\right) e^{-i \omega_{j}\left(t-t^{\prime}\right)} d t^{\prime},
$$

where the first term is the free evolution of the bath operator, while the second term is arising due to the interaction with the system. Inserting Eq. (7) into Eq. (5), we find

$$
\begin{aligned}
& \dot{\hat{a}}(t)=-i \omega_{0} \hat{a}(t)+(n+1) \sum_{k} g_{k} \hat{b}_{k}\left(\hat{a}^{\dagger}\right)^{n}(t) e^{-i \omega_{k} t} \\
& -(n+1) \sum_{k} g_{k}^{2}\left(\hat{a}^{\dagger}\right)^{n}(t) \int_{0}^{t} \hat{a}^{n+1}\left(t^{\prime}\right) e^{-i \omega_{k}\left(t-t^{\prime}\right)} d t^{\prime}
\end{aligned}
$$

Now introducing slowly varying operator $\hat{A}(t)=\hat{a}(t) e^{i \omega_{0} t}$ in Eq. (8), which varies little over the inverse reservoir bandwidth, we can take the system operator out of the integral by substituting $\hat{A}\left(t^{\prime}\right) \simeq \hat{A}(t)$, under Markov approximation [2]. Then replacing the remaining integral of $t^{\prime}$ by the usual $\delta$-function [6], we finally arrive at the reduced operator equation for the system which is given by

$$
\dot{\hat{A}}(t)=-\gamma_{n+1}\left(\hat{A}^{\dagger}\right)^{n}(t) \hat{A}^{n+1}(t)+\hat{F}_{n+1}(t)\left(\hat{A}^{\dagger}\right)^{n}(t),
$$

and its hermitian adjoint. The reduced dynamics described by the operator Langevin Eq. [9], contains the usual dissipative term

$$
\gamma_{n+1}=(n+1) \sum_{k} g_{k}^{2} \pi \delta\left(\omega_{k}-(n+1) \omega_{0}\right),
$$

as well as the noise term

$$
\hat{F}_{n+1}(t)=(n+1) \sum_{k} g_{k} \hat{b}_{k} \exp \left[-i\left\{\omega_{k}-(n+1) \omega_{0}\right\} t\right],
$$

which is multiplicative in nature. The noise operator in Eq. (9) appears as a natural consequence of the systemreservoir coupling. It is thus imperative that the Liénard system with a microscopic basis must be internally noisy. Combining Eq. (9) and its hermitian adjoint we may further define a noise operator $\hat{G}_{n+1}(t)=\hat{F}_{n+1}(t)+\hat{F}_{n+1}^{\dagger}(t)$.

The properties of the reservoir can then be calculated by thermal averaging over appropriately ordered noise operators. To this end we define quantum statistical average of any reservoir operator $\hat{O}$ as

$$
\langle\hat{O}(t)\rangle_{q s}=\frac{\operatorname{Tr}\left[\hat{O} \exp \left(-\hat{H}_{R} / K T\right)\right]}{\operatorname{Tr}\left[\exp \left(-\hat{H}_{R} / K T\right)\right]},
$$

where $\hat{H}_{R}=\sum_{j} \hbar \omega_{j} \hat{n}_{j}$ at $t=0$ and $\hat{n}_{j}$ denotes the number operator for the $j$-th bath mode. 
Based on the above considerations, the noise properties of the operator may be calculated by using the canonical thermal distribution Eq. (11). It can be shown that the noise is zero-centered, so that

$$
\left\langle\hat{G}_{n+1}(t)\right\rangle_{q s}=0
$$

and satisfies the following relation

$$
\begin{aligned}
& \operatorname{Re}\left[\left\langle\hat{F}_{n+1}^{\dagger}(t) \hat{F}_{n+1}\left(t^{\prime}\right)+\hat{F}_{n+1}(t) \hat{F}_{n+1}^{\dagger}\left(t^{\prime}\right)\right\rangle_{q s}\right] \\
& =(n+1)^{2} \sum_{k} g_{k}^{2}\left\langle\left(2 \hat{n}_{k}^{B}+1\right)\right\rangle_{q s} \cos \left\{\left[\omega_{k}-(n+1) \omega_{0}\right]\left(t-t^{\prime}\right)\right\}
\end{aligned}
$$$$
=(n+1)^{2} \sum_{k} g_{k}^{2} \operatorname{coth}\left(\frac{\hbar \omega_{k}}{2 K T}\right) \cos \left\{\left[\omega_{k}-(n+1) \omega_{0}\right]\left(t-t^{\prime}\right)\right\}
$$

where the cotangent hyperbolic factor in Eq. (14) can be identified with the Bose-Einstein distribution

$$
\left\langle\hat{n}_{k}^{B}\right\rangle_{q s}=\frac{1}{e^{\hbar \omega_{k} / K T}-1}=\bar{n}_{B}\left(\omega_{k}\right)
$$

using the following relation

$$
\left\langle\left(2 \hat{n}_{k}^{B}+1\right)\right\rangle_{q s}=2 \bar{n}_{B}\left(\omega_{k}\right)+1=\operatorname{coth}\left(\frac{\hbar \omega_{k}}{2 K T}\right) .
$$

Equation (14) refers to the fluctuation-dissipation relation for a bosonic bath, which guarantees that the overall system is thermodynamically closed. In the subsequent sections we show that the above detailed balance helps us to preserve the dynamical stability of the limit cycle even in presence of noise. An external noise of even very weak intensity on the other hand destroys the attractors. The plus one factor in Eq. (16) is responsible for vacuum fluctuation which is always present at quantum scale even at absolute zero. Its implication on the microscopic realization of limit cycles will be analyzed in the following sections.

\section{C-NUMBER DESCRIPTION OF QUANTUM LANGEVIN EQUATION}

The main purpose of this section is to construct a quantum Langevin equation with c-number noise. As a first step, we return to Eq. (9) and carry out the quantum mechanical average $\langle\ldots\rangle$ over the initial product separable quantum states of the system oscillator and the bath oscillators at $t=0,|\alpha\rangle\left|\mu_{1}\right\rangle\left|\mu_{2}\right\rangle \ldots\left|\mu_{k}\right\rangle \ldots\left|\mu_{N}\right\rangle$ to obtain

$$
\langle\dot{\hat{A}}(t)\rangle=-\gamma_{n+1}\left\langle\left(\hat{A}^{\dagger}\right)^{n}(t) \hat{A}^{n+1}(t)\right\rangle+\left\langle\hat{F}_{n+1}(t)\right\rangle\left\langle\left(\hat{A}^{\dagger}\right)^{n}(t)\right\rangle .
$$

Here $|\alpha\rangle$ refers to the initial coherent state of the system and $\left\{\left|\mu_{k}\right\rangle\right\}$ correspond to initial coherent states of the bath operators. The rationale behind using harmonic oscillator coherent states for the system and bath operators is to recast Eq. 17 as classical-looking Langevin equation for the reduced system oscillator interacting with a bosonic bath.

Defining the following quantum mechanical averages for the system and noise operators

$$
\langle\hat{A}(t)\rangle=\alpha(t) ;\left\langle\hat{A}^{\dagger}(t)\right\rangle=\alpha^{*}(t) ;\left\langle\hat{G}_{n+1}(t)\right\rangle=\xi_{n+1}(t),
$$

Eq. 17) and its adjoint can be written as

$$
\begin{aligned}
\dot{\alpha}(t) & =-\gamma_{n+1}|\alpha|^{2 n} \alpha+f_{n+1}(t)\left(\alpha^{*}\right)^{n}, \\
\dot{\alpha}^{*}(t) & =-\gamma_{n+1}|\alpha|^{2 n} \alpha^{*}+f_{n+1}^{*}(t) \alpha^{n} .
\end{aligned}
$$

Here the c-number quantum noise $\xi_{n+1}(t)$ is given by

$$
\begin{aligned}
\xi_{n+1}(t)= & f_{n+1}(t)+f_{n+1}^{*}(t), \\
= & (n+1) \sum_{k} g_{k}\left[\mu_{k}(0) \exp \left\{-i\left(\omega_{k}-(n+1) \omega_{0}\right) t\right\}\right. \\
& \left.+\mu_{k}^{*}(0) \exp \left\{i\left(\omega_{k}-(n+1) \omega_{0}\right) t\right\}\right],
\end{aligned}
$$

where $\mu_{k}$ and $\mu_{k}^{*}$ are the associated c-numbers for the bath operators. In deriving Eqs. (19) from Eq. (17), we only consider normal ordering for the system operators. Instead for use of different ordering of the operators, we may end up with altogether different (but equivalent [2]) forms for the nonlinear damping. We will return to this point in the next section when we discuss the quantumclassical correspondance for the limit cycles within the framework of the same interaction Hamiltonian under mean-field approximation [17-19].

Now to realize $\xi_{n+1}(t)$ as an effective c-number noise, we introduce the ansatz that $\mu_{k}(0)$ and $\mu_{k}^{*}(0)$ in Eq. 20 are distributed according to Wigner thermal canonical distribution of Gaussian form [20] as follows

$$
W_{k}^{B}\left[\mu_{k}(0), \mu_{k}^{*}(0)\right]=N_{B} \exp \left\{-\frac{\left|\mu_{k}(0)\right|^{2}}{2 \operatorname{coth}\left(\frac{\hbar \omega_{k}}{2 K T}\right)}\right\} .
$$

Here $N_{B}$ is the normalization constant and $\operatorname{coth}\left(\frac{\hbar \omega_{k}}{2 K T}\right)$ is the width of the distribution. For any arbitrary quantum mechanical mean value of a bath operator $\left\langle\hat{B}_{k}\right\rangle$, which is a function of $\mu_{k}(0)$ and $\mu_{k}^{*}(0)$, its statistical average can then be calculated as

$$
\left\langle\left\langle\hat{B}_{k}\right\rangle\right\rangle_{s}=\int\left\langle\hat{B}_{k}\right\rangle W_{k}^{B}\left[\mu_{k}(0), \mu_{k}^{*}(0)\right] d \mu_{k}(0) d \mu_{k}^{*}(0) .
$$

Using the ansatz of Eq. (21) and definition of statistical average Eq. (22), one can show that the c-number noise $\xi_{n+1}(t)$ satisfies the following relations, respectively,

$$
\left\langle\xi_{n+1}(t)\right\rangle_{s}=0
$$

and

$$
\begin{aligned}
& \left\langle\xi_{n+1}(t) \xi_{n+1}^{*}\left(t^{\prime}\right)\right\rangle_{s} \\
& =(n+1)^{2} \sum_{k} g_{k}^{2} \operatorname{coth}\left(\frac{\hbar \omega_{k}}{2 K T}\right) \cos \left\{\left(\omega_{k}-(n+1) \omega_{0}\right)\left(t-t^{\prime}\right)\right\} .
\end{aligned}
$$


Equations 23 and 24 imply that the c-number noise $\xi_{n+1}(t)$ is zero-centered and follows the fluctuationdissipation relation as expressed in Eq. (14). Therefore, Eqs. (24) and (14) are equivalent. In order to identify the connection between Eqs. (14) and 24, it is sometime convenient to write down the fluctuation-dissipation relation in the following form:

$$
\begin{aligned}
& \left\langle\xi_{n+1}(t) \xi_{n+1}^{*}\left(t^{\prime}\right)\right\rangle_{s} \\
& =\operatorname{Re}\left[\left\langle\hat{F}_{n+1}^{\dagger}(t) \hat{F}_{n+1}\left(t^{\prime}\right)+\hat{F}_{n+1}(t) \hat{F}_{n+1}^{\dagger}\left(t^{\prime}\right)\right\rangle_{q s}\right] \\
& =2(n+1) \gamma_{n+1} \operatorname{coth}\left[\frac{(n+1) \hbar \omega_{0}}{2 K T}\right] \delta\left(t-t^{\prime}\right)
\end{aligned}
$$

In deriving the above expression we have assumed that the bath modes are closely spaced in frequency so that one may replace the summation over $k$ in Eq. (24) by an integral over $\omega$ using the density of states $\rho(\omega)$ [6] which yields $\gamma_{n+1}=(n+1) \pi g^{2}\left((n+1) \omega_{0}\right) \rho\left((n+1) \omega_{0}\right)$. Secondly, Eq. 25 depends on the noise operator ordering, but not on time ordering. This indicates a clear-cut advantage of the c-number formalism which allows us to bypass the operator ordering prescription for the derivation of noise properties [21]. The c-number noise $\xi_{n+1}(t)$ as characterized by Eqs. 23) and (24) is classical-looking in form but essentially quantum mechanical in nature. Therefore, the essence of microscopic origin of quantum limit cycles can be captured in the present formalism quite effectively in terms of c-number description by simply implementing the techniques of classical non-equilibrium statistical mechanics. Above formalism has been extensively used in several earlier occasions in connection with quantum optics, chemical dynamics, and multidimensional transition state theory in the context of spin and bosonic baths [21 23].

\section{CONSTRUCTION OF QUANTUM LIÉNARD SYSTEM; APPLICATION OF THE PROPOSED MODEL}

We are now in a position to apply our method to various nonlinear systems that produce limit cycle oscillations. Two such classic examples are Van der Pol and Rayleigh oscillators, whose basic equation of motions are

$$
\ddot{x}+\omega_{0}^{2} x-\epsilon\left(1-x^{2}\right) \dot{x}=0
$$

and

$$
\ddot{x}+\omega_{0}^{2} x-\epsilon\left(1-\dot{x}^{2}\right) \dot{x}=0
$$

respectively, where we assume $\epsilon>0$. A broad class of biological and chemical oscillations are modelled either in terms of Rayleigh or Van der Pol oscillator or in terms of their generalization [24 26]. According to Eq. (4), both the above models are subject to internal quantum noise satisfying fluctuation-dissipation theorem, when derived from their respective microscopic Hamiltonians. The form of the interaction for these specific examples simplifies to

$$
\hat{H}_{I}=i \hbar \sum_{k} g_{k}\left[\left(\hat{a}^{\dagger}\right)^{2} \hat{b}_{k}-(\hat{a})^{2} \hat{b}_{k}^{\dagger}\right]
$$

which is a special case of Eq. (2). From this interaction Hamiltonian [Eq. (28)], we proceed as in Sec. II and finally arrive at the following operator Langevin equations for the reduced system

$$
\dot{\hat{A}}(t)=-\gamma_{2} \hat{A}^{\dagger}(t) \hat{A}^{2}(t)+\hat{F}_{2}(t) \hat{A}^{\dagger}(t)
$$

which is the generalized operator Eq. $(9)$ for $n=1$. If we follow the usual normal ordering prescription discussed in Sec. III the resulting quantum dynamics becomes identical to the generalized c-number Eq. 19 with $n=1$ :

$$
\dot{\alpha}(t)=-\gamma_{2}|\alpha|^{2} \alpha+f_{2}(t) \alpha^{*}
$$

Substituting $\alpha$ and $\alpha^{*}$ by

$$
\begin{gathered}
\alpha=\frac{1}{2}\left(x-\frac{i \dot{x}}{\omega_{0}}\right) \exp \left[-i \omega_{0} t\right], \\
\alpha^{*}=\frac{1}{2}\left(x+\frac{i \dot{x}}{\omega_{0}}\right) \exp \left[i \omega_{0} t\right]
\end{gathered}
$$

and collecting the terms of right order from Eq. (30), we have the following differential equation for the system

$$
\ddot{x}+\gamma_{2} x^{2} \dot{x}+\omega_{0}^{2} x=\eta_{2}(x, \dot{x}),
$$

where

$$
\begin{aligned}
\eta_{2}(x, \dot{x})= & \frac{i \omega_{0}}{2}\left[f_{2}\left(x+\frac{i \dot{x}}{\omega_{0}}\right) e^{2 i \omega_{0} t}\right. \\
& \left.-f_{2}^{*}\left(x-\frac{i \dot{x}}{\omega_{0}}\right) e^{-2 i \omega_{0} t}\right],
\end{aligned}
$$

is the quantum noise term arising out of the systemreservoir interaction. Equation (32) may be regarded as the simplest representation of the quantum Liénard system [Eq. (4)] where $f(x)=\epsilon x^{2}$ and $g(x)=\omega_{0}^{2} x$. The typical phase portraits of the system in absence and presence of internal noise are shown in Fig. 1. We use python code for random number generators [27] to simulate the Gaussian white noise 28]. Throughout our paper we choose $\omega_{0}=1$ for numerical calculations. In presence of noise, the stochastic trajectories neither collapse to a steady state nor diverge. This implies that the detailed balance in the form of fluctuation-dissipation [Eq. (24)] guarantees the dynamical stability of motion [Fig. 1 b] If the detailed balance in the form of Eq. 24 is not properly maintained, the limit cycle is destroyed. This is illustrated in [Fig. 1 c] when the noise is of external origin. 

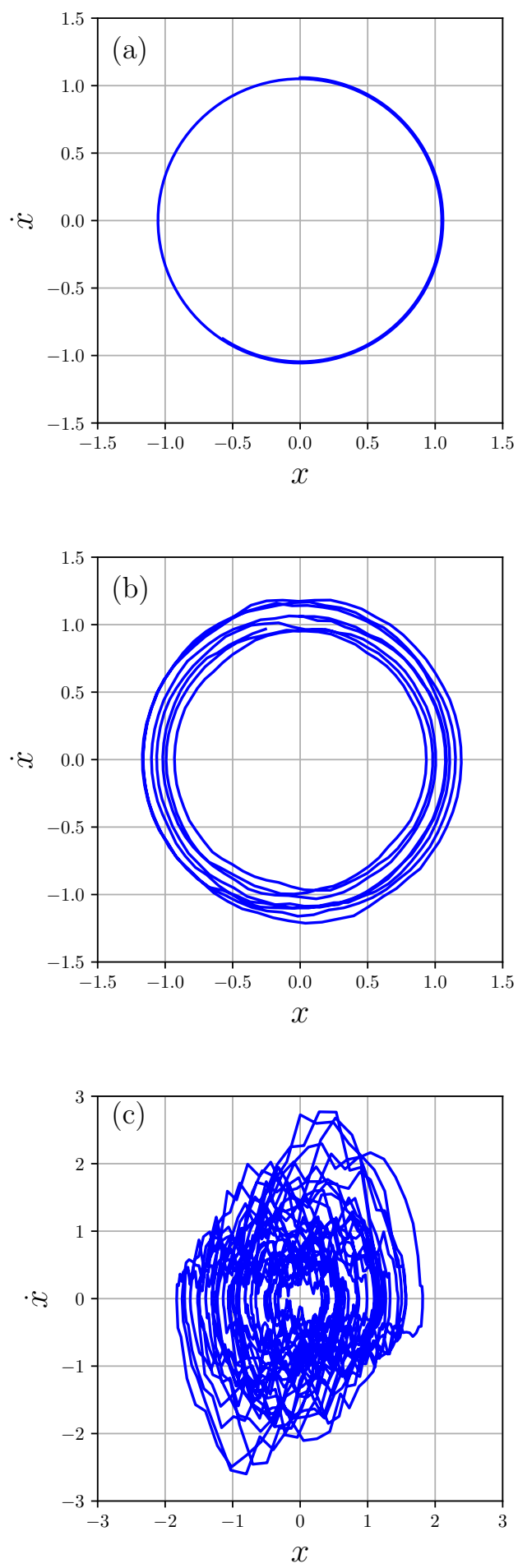

FIG. 1. The phase space trajectories of (a) classical and (b) quantum Liénard oscillator [Eq. (32)] with $f(x)=\epsilon x^{2}$ are plotted in absence (a) and presence (b) of intrinsic noise. (c) Quantum Liénard oscillator in presence of external noise: lack of detailed balance destroys the limit cycle oscillation. We set $\epsilon=0.01$ and $K T=50$ (units arbitrary).

Equations (29) (and (30) are the quantum Liénard equation in operator (and c-number) form. In order to recover the two special cases of this system we proceed as follows: we first note that the use of commutation relation $\left[\hat{A}, \hat{A}^{\dagger}\right]=1$ in the operator Eq. (29) leads to several other equivalent forms [2]. For example, we have for Eq. 29.

$$
\dot{\hat{A}}(t)=-\gamma_{2}\left(\hat{A} \hat{A}^{\dagger} \hat{A}-\hat{A}\right)+\hat{F}_{2}(t) \hat{A}^{\dagger}(t) .
$$

More generally we may write for Eq. (9)

$$
\begin{aligned}
\dot{\hat{A}}(t)= & -\gamma_{n+1} \sum_{p, q \ldots r} \phi(p, q . . r) \hat{A}^{p}\left(\hat{A}^{\dagger}\right)^{q} \ldots \hat{A}^{r} \\
& +\hat{F}_{n+1}(t)\left(\hat{A}^{\dagger}\right)^{n}(t) .
\end{aligned}
$$

In the next step we derive the c-number equivalent of the above operator equation such that it corresponds to the specific classical Liénard form. This may be achieved by appropiate operator ordering followed by performing average with coherent states under mean field approximation. We are then led a desired form of c-number equivalent of Eq. 35

$$
\dot{\alpha}=-\gamma_{n+1} \sum_{i, j} \psi(i, j) \alpha^{i}\left(\alpha^{*}\right)^{j}+f_{n+1}(t)\left(\alpha^{*}\right)^{n} .
$$

A pertinent point is to be noted. Recognizing the right hand side of Eq. (36) as a polynomial in c-numbers, the term with its highest power is sufficient for the existance of limit cycle as shown in Eq. (30). The inclusion of terms of lower power is necessary to retain the shape of the limit cycle that conforms faithfully with its classical counterpart. The mean field approximation therefore allows us to establish a quantum-classical correspondence. Furthermore we emphasize that the stability of the limit cycles and their number remain invariant with respect to the inclusion of terms with lower power of the polynomial. Based on these considerations Eq. (34) takes the following form (For details see Appendix)

$$
\dot{\alpha}=-\frac{\gamma_{2}}{m_{1}}\left(m_{1}|\alpha|^{2}+m_{0}\right) \alpha+f_{2} \alpha^{*},
$$

which may correspond to the specific Liénard system for specific values of $m_{1}$ and $m_{0}$. In what follows, we will show that Van der Pol and Rayleigh oscillators appear from it as special cases.

\section{A. Van der Pol oscillator}

The Van der Pol oscillator is a prototypical selfsustained oscillator which has been used to model the dynamics of a variety of classical [29] and biological processes, such as heart [30, neurons [31], and circadian rhythms [32]. If we substitute $m_{1}=1$ and $m_{0}=-1$ in Eq. (37), we find

$$
\dot{\alpha}(t)=-\gamma_{2}\left(|\alpha|^{2}-1\right) \alpha+f_{2}(t) \alpha^{*},
$$


and its complex conjugate, where the c-number noise expression follows from Eq. (20) as

$$
f_{2}=2 \sum_{k} g_{k} \mu_{k}(0) \exp \left[-i\left(\omega_{k}-2 \omega_{0}\right) t\right]
$$

If we further replace $\alpha$ and $\alpha^{*}$ in Eq. 38 by the usual form [Eq. (31)] and neglect the high frequency terms, one may obtain the following differential equation after little bit of rearrangement

$$
\ddot{x}+\gamma_{2}\left(x^{2}-1\right) \dot{x}+\omega_{0}^{2} x=\eta_{2}(x, \dot{x}),
$$

where by definition $\gamma_{2}>0$.
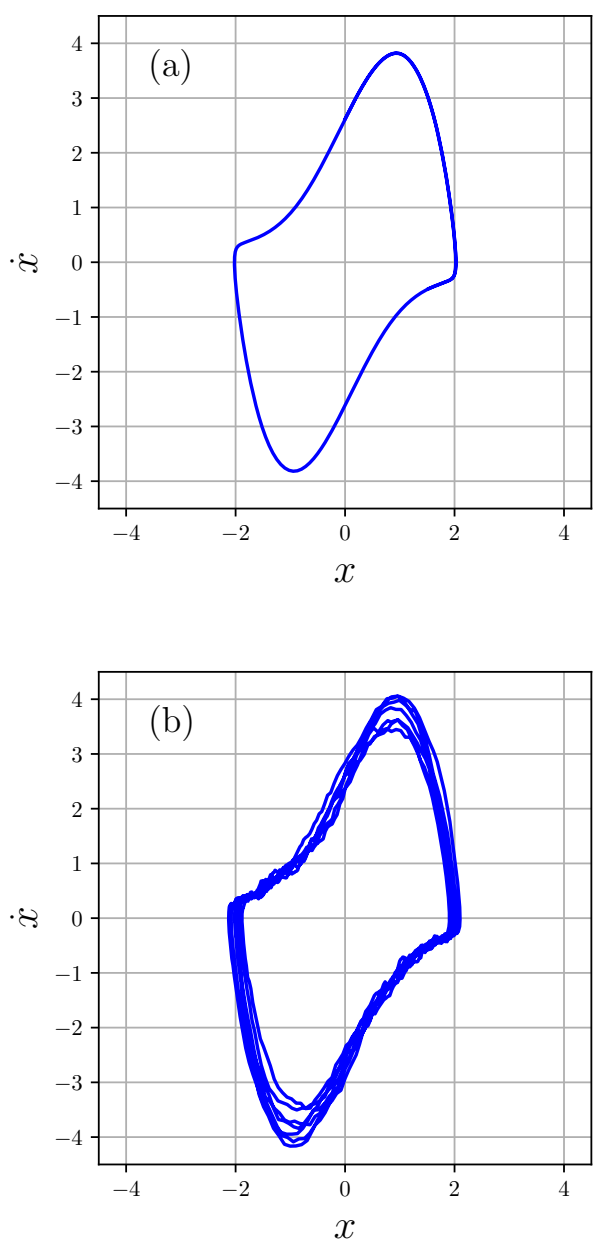

FIG. 2. (a) The phase space trajectories of a (a) classical [Eq. (26)] and (b) quantum Van der Pol oscillator [Eq. (40]] are plotted for $\epsilon=2$ and $K T=0$. It is shown that quantum fluctuations can retain the general shape of the limit cycle oscillation even at absolute zero (units arbitrary).

The c-number Eq. (40) looks similar to classical equation of Van der Pol oscillator which is simultaneously driven by the c-number quantum noise. This quantum Van der Pol oscillator can therefore be regarded as a quantum harmonic oscillator with two types of dissipation: negative $(-\dot{x})$ as well as nonlinear $\left(x^{2} \dot{x}\right)$ damping, and an internal noise - combination of which leads to a noisy but self-sustained limit cycle oscillation. The presence of limit cycle suggests that for the system which follows the isolated asymptotic trajectory, the gain in energy due to self-excitation is equal to the loss of energy due to dissipation. The fluctuation-dissipation relation on the other hand entails the detailed balance condition which implies that the energy dissipated by the system on an average is equal to the energy gained by it through fluctuation of the reservoir. The energy loss-gain in the former processes is purely dynamical while that as a result of detailed balance is statistical in nature.

Because of its simple form, such quantum Van der Pol oscillator has recently gained significant interest for realization of synchronization phenomena at quantum scale [33-36]. Interestingly, experiment with trapped-ions may serve as an ideal test bed for simulating collective dynamics for such oscillators [37-40]. The phase space diagrams for both classical van der Pol oscillator and its quantum counterpart are plotted in Fig. 2 for illustration.

\section{B. Rayleigh oscillator}

Similar to Van der Pol oscillator, Rayleigh oscillator can also be obtained from same interaction Hamiltonian Eq. 28 or in other words from the same generalized cnumber Langevin dynamics [Eq. (37)]. This immediately suggests that both the noisy Van der Pol and Rayleigh oscillators are equivalent to the simplest possible form of Liénard system [Eq. (30]] within a mean-field description. Thus the characteristic features of both the two oscillators can be essentially captured by Eq. (32).

To recover the explicit form of Rayleigh oscillator, we substitute $m_{1}=3$ and $m_{0}=-1$ in Eq. (37), so that we get

$$
\dot{\alpha}(t)=\frac{\gamma_{2}}{3}\left(1-3|\alpha|^{2}\right) \alpha+f_{2} \alpha^{*},
$$

and its complex conjugate. To arrive at the standard form of the Rayleigh oscillator equation from Eq. 41, instead of Eq. (31), we now use the following substitution for $\alpha$ and $\alpha^{*}$,

$$
\begin{aligned}
\alpha & =\frac{1}{2}\left(\omega_{0} x-i \dot{x}\right) \exp \left[-i \omega_{0} t\right], \\
\alpha^{*} & =\frac{1}{2}\left(\omega_{0} x+i \dot{x}\right) \exp \left[+i \omega_{0} t\right] .
\end{aligned}
$$

This difference in the substitution can be traced back to the dimensional relationship between the phase space dynamical variables of the two oscillators as understandable from Eqs. 26) and (27). With the help of Eqs. (42) and using the same treatment as before, we finally reduce the following equation of motion for the quantum Rayleigh oscillator

$$
\ddot{x}+\frac{1}{3} \gamma_{2}\left(\dot{x}^{2}-1\right) \dot{x}+\omega_{0}^{2} x=\eta_{2}(x, \dot{x}) .
$$



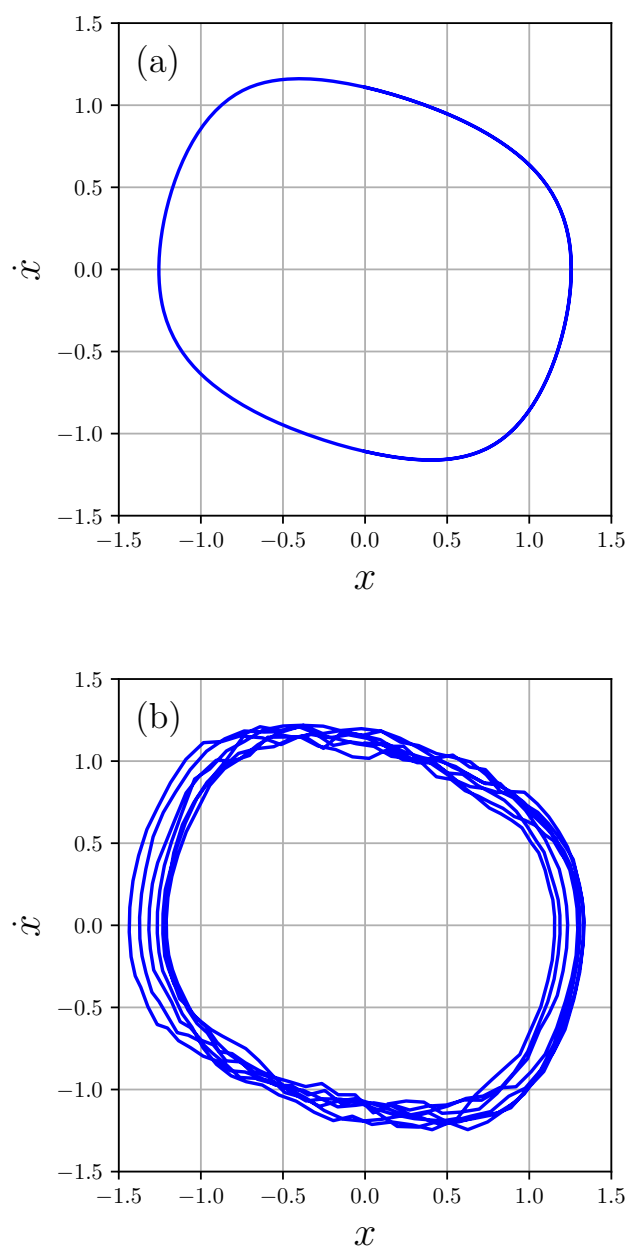

FIG. 3. (a) The phase space trajectories of a (a) classical [Eq. (27)] and (b) quantum Rayleigh oscillator [Eq. [43] ] are plotted for the parameters $\epsilon=1$ and $K T=10$ (units arbitrary).

Similar to Van-der Pol oscillator, Rayleigh oscillator plays a close kinship with parametric oscillation in pipe organs, as pioneered by Rayleigh [41] and in wave mixing in nonlinear optics [42]. The phase space plots for classical Rayleigh oscillator and its quantum version are depicted in Fig. 3 In general, we find that average quantum dynamics agrees with the phase boundary of the classical limit cycle even at the microscopic scale.

\section{Construction of generalized quantum Liénard oscillators}

Let us now extend our formalism to construct a broader class of Liénard oscillators. For this we adopt the following recipe: First we resort to operator Langevin Eq. (9) and discuss the first two cases $[n=1$ and 2 of Eq. (9)] as illustrative examples. Next we generalize the concept(see Appendix for details) to construct the most generic form of the Liénard oscillators that can be derived from our model Hamiltonian Eq. (2).

- Case-1: $f(x)=\epsilon\left(a_{1} x^{2}+a_{0}\right)$ :- In the previous section we have shown that a general form of $f(x)=$ $\epsilon\left(a_{1} x^{2}+a_{0}\right)$ can be obtained from the interaction Hamiltonian $\hat{H}_{I}=i \hbar \sum_{k} g_{k}\left[\left(\hat{a}^{\dagger}\right)^{2} \hat{b}_{k}-(\hat{a})^{2} \hat{b}_{k}^{\dagger}\right]$. The respective c-number Langevin dynamics is given by Eq. (37) and the corresponding equation of motion takes the form of $\ddot{x}+\omega_{0}^{2} x=-\left(\gamma_{2} / m_{1}\right)\left(m_{1} x^{2}+m_{0}\right) \dot{x}+\eta_{2}(x, \dot{x})$, where we may choose $m_{1}$ and $m_{0}$ so that it is consistent with the values $a_{1}, a_{0}$ and $\epsilon$ of $f(x)$. Essentially one sets $\epsilon=\left(\gamma_{2} / m_{1}\right)$, then $m_{1}$ and $m_{0}$ become identical to $a_{1}$ and $a_{0}$ respectively. This can be regarded as a representation of Liénard system in quantum scenario with the friction coefficient $f(x)=\epsilon\left(a_{1} x^{2}+a_{0}\right)$, such that our desired system exhibits a single quantum limit cycle.

- Case-2: $f(x)=\epsilon\left(a_{2} x^{4}+a_{1} x^{2}+a_{0}\right)$ :- Let us now consider most general form of $f(x)$ with the next highest order power in accordance with the Liénard's theorem. In the similar spirit one can show that the above form of $f(x)$ can be obtained from the Hamiltonian

$$
\hat{H}_{I}=i \hbar \sum_{k} g_{k}\left[\left(\hat{a}^{\dagger}\right)^{3} \hat{b}_{k}-(\hat{a})^{3} \hat{b}_{k}^{\dagger}\right]
$$

which corresponds to $n=2$ of the generalized interaction Hamiltonian [Eq. (2)]. From the operator Langevin equations Eq. (9), one carry out the same procedure to derive the c-number Langevin equations for $n=2$ (see Appendix)

$$
\dot{\alpha}=-\frac{\gamma_{3}}{m_{2}}\left[m_{2}|\alpha|^{4}+m_{1}|\alpha|^{2}+m_{0}\right] \alpha+f_{3}\left(\alpha^{*}\right)^{2} .
$$

Following the standard substitution as given in Eq. (31) we find

$$
\ddot{x}+\omega_{0}^{2} x=-\frac{\gamma_{3}}{m_{2}}\left[\frac{m_{2}}{2} x^{4}+m_{1} x^{2}+m_{0}\right] \dot{x}+\eta_{3}(x, \dot{x}) .
$$

TABLE I.

\begin{tabular}{ccccc}
\hline \hline Ex. & $m_{2}$ & $m_{1}$ & $m_{0}$ & $f(x)$ \\
\hline 1 & 2 & 1 & -1 & $\epsilon\left(x^{4}+x^{2}-1\right)$ \\
2 & 2 & -1 & 0 & $\epsilon\left(x^{4}-x^{2}\right)$ \\
3 & 2 & 0 & -1 & $\epsilon\left(x^{4}-1\right)$ \\
\hline
\end{tabular}

This is the relevant quantum version of Liénard system with $f(x)=\epsilon\left(a_{2} x^{4}+a_{1} x^{2}+a_{0}\right)$, where we have to choose the values of $m_{2}, m_{1}$ and $m_{0}$ judiciously, such that it matches with the known form of $f(x)$. Some of the choices of $\left\{m_{2}, m_{1}, m_{0}\right\}$ are shown in the Table I Note that in each case the value of $\epsilon$ varies as $\gamma_{3} / m_{2}$. It was 
shown by Rychkov 43 that the number of limit cycles is at most two for $f(x)=\epsilon\left(a_{2} x^{4}+a_{1} x^{2}+a_{0}\right)$. Now extrapolating the form of the above two cases we may generalize the formula by the method of mathematical induction to an arbitrary polynomial $f(x)$ as given below:

- Case-3: $f(x)=a_{n} x^{2 n}+a_{n-1} x^{2 n-2} \ldots+a_{1} x^{2}+a_{0}$ :Let us now consider the most general form of an even function $f(x)$. From the operator equation of motion (9) one can evaluate the following equations for the cnumber Langevin dynamics (see Appendix)

$$
\begin{aligned}
\dot{\alpha}= & -\frac{\gamma_{n+1}}{m_{n}}\left[m_{n}|\alpha|^{2 n}+m_{n-1}|\alpha|^{2 n-2} \ldots .+m_{1}|\alpha|^{2}\right. \\
& \left.+m_{0}\right] \alpha+f_{n+1}\left(\alpha^{*}\right)^{n} \\
\dot{\alpha}^{*}= & -\frac{\gamma_{n+1}}{m_{n}}\left[m_{n}|\alpha|^{2 n}+m_{n-1}|\alpha|^{2 n-2} \ldots .+m_{1}|\alpha|^{2}\right. \\
& \left.+m_{0}\right] \alpha^{*}+f_{n+1}^{*} \alpha^{n},
\end{aligned}
$$

where $m_{0}, m_{1}, \ldots m_{n}$ are independent of one another. Proceeding in the similar way, it is possible to construct the following dynamical equation of motion for the system

$$
\begin{aligned}
\ddot{x}+\omega_{0}^{2} x= & -\frac{\gamma_{n+1}}{m_{n}}\left(a_{n} x^{2 n}+a_{n-1} x^{2 n-2} \ldots .+a_{1} x^{2}+a_{0}\right) \dot{x} \\
& +\eta_{n+1}(x, \dot{x}),
\end{aligned}
$$

where

$$
a_{j}=\frac{m_{j}}{{ }^{2 j} C_{j+1}} j, \quad \forall \quad j=1,2 \ldots n \quad \text { and } \quad a_{0}=m_{0} .
$$

Above equation gives us the relation between different $m_{j}$ 's with $a_{j}$ 's. Note that for $n=1$ (Case-1), $a_{j}=m_{j}$ irrespective of all $j$.

According to Refs. [16, 44, 46] the system of LiénardSmith-Levinson form with $f(x)$, a polynomial of highest degree $2 n$ where the coefficients, $a_{n}, a_{n-1} \ldots a_{1}$ alternate in sign, can support at most $n$ number of limit cycles. The Eq. (49) can be interpreted as quantum generalization of Liénard oscillator with most general form of $f(x)$ which may have at most $n$ number of limit cycles. Phase portraits of a multiple limit cycle system in both classical and quantum scenario are plotted in Fig. 4. Classically, any noisy limit cycle at $T=0$ does not make any sense, but quantum mechanically, due to vacuum fluctuation one can have stable limit cycle oscillation even at absolute zero. This is a pure quantum phenomenon which does not have any classical analog. As an extension of Liénard-Smith-Levinson form of oscillators, generalized Rayleigh family of oscillators [13, 47, 48] can also be constructed from the above prescription (see Appendix).
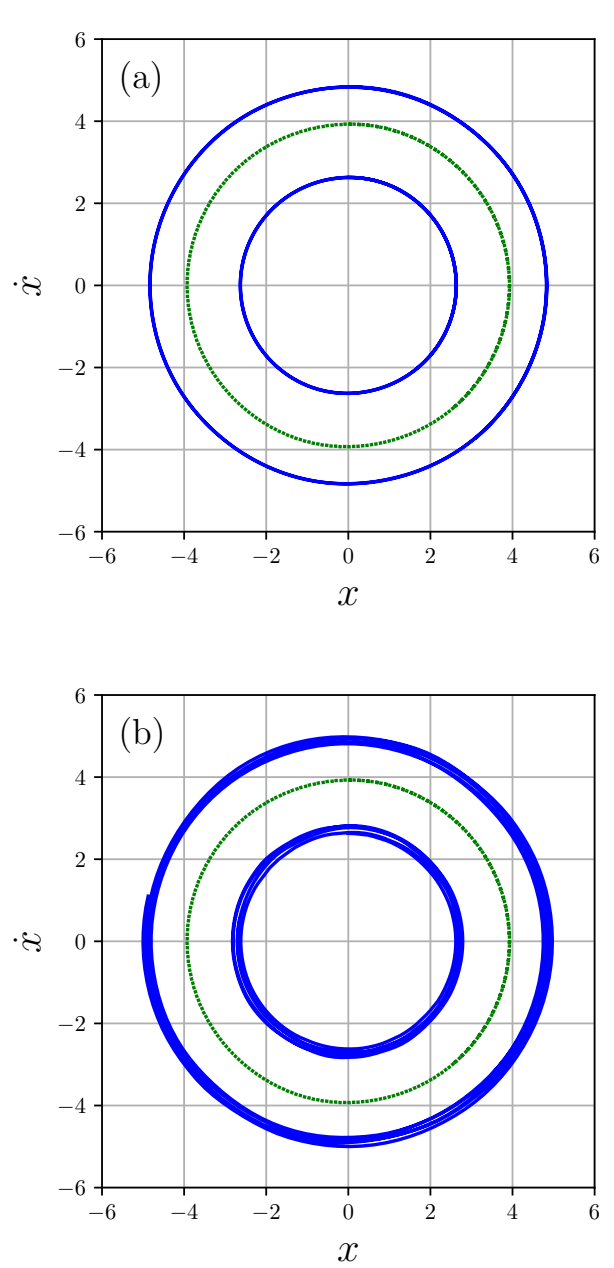

FIG. 4. The phase space trajectories of (a) classical and (b) quantum Liénard system with $f(x)=0.005 * x^{6}-0.144 * x^{4}+$ $x^{2}-1$ [16] are plotted for $\epsilon=0.01$. In both cases we have two stable (blue line) and one unstable (green dotted line) limit cycles. Pure quantum fluctuations at $T=0$ may lead to noisy but stable oscillations even for the two stabe limit cycles (units arbitrary).

\section{CONCLUSIONS}

In this work we have presented a generalized microscopic quantum description of classical Liénard system. Our approach is based on two essential elements. First, the interaction Hamiltonian consisting of suitable nonlinear coupling terms serves as a universal paradigm for description of several variants of Liénard system with appropriate noise and nonlinear dissipation. Second, the harmonic oscillator coherent states facilitate the use of c-number description for reduced oscillator equation of motion. The major findings of our work are as follows:

(i) A classical Liénard system without any noise is purely a nonlinear dynamical oscillator which depending on the specificity of nonlinear dissipation admits of 
single or multiple limit cycles. As opposed to it, quantum noise appears in the dynamics as a consequence of system-reservoir framework of our microscopic model and carries the signature of pure quantum effects.

(ii) The effect of quantum noise makes the stable limit cycle fuzzy; however, it does not loose its stability in the sense that the limit cycle trajectory neither collapses to a steady state nor diverges. The noninterference of the two energy balance processes is characteristic of the internal noise of a thermodynamically closed system. Thus the detailed balance through nonlinear fluctuationdissipation relation keeps the dynamical stability of the limit cycle preserved. In contrast, the external noise tends to destroy the limit cycle or, in general, the dynamical stability of the Liénard system.

(iii) The Van der Pol and Rayleigh oscillators, though behave distinctly at the classical level, are identical at the microscopic level within a mean-field description. In fact a large number of Liénard systems (both Van der Pol and Rayleigh families of cycles) differing in the form of their nonlinear damping can be constructed from the same microscopic Hamiltonian at the mean-field level. In all such cases the number of limit cycles is determined by the highest power of the polynomial governing the nonlinear dissipation.

\section{ACKNOWLEDGEMENTS}

S. B. thanks DST INSPIRE, A. G. thanks Initiation Grant, IITK and SRG, SERB, India and D.S.R thanks DST, under Government of India, for a J. C. Bose National Fellowship under Grant No. SB/S2/JCB-030/2015 for partial financial support.
[1] K. Lindenberg and B. West, The Nonequilibrium Statistical Mechanics of Open and Closed Systems (VCH Publishers, 1990); U. Weiss, Quantum Dissipative Systems, Series in modern condensed matter physics (World Scientific, 1999).

[2] W. Louisell, Quantum Statistical Properties of Radiation, A Wiley-Interscience publication (Wiley, 1973); H. Carmichael and S.-V. (Berlin)., Statistical Methods in Quantum Optics 1: Master Equations and FokkerPlanck Equations, Physics and astronomy online library (Springer, 1999).

[3] R. Zwanzig, Journal of Statistical Physics 9, 215 (1973)

[4] A. O. Caldeira and A. J. Leggett, Phys. Rev. Lett. 46, 211 (1981) A. Caldeira and A. Leggett, Annals of Physics 149, 374 (1983) A. J. Leggett, S. Chakravarty, A. T. Dorsey, M. P. A. Fisher, A. Garg, and W. Zwerger, Rev. Mod. Phys. 59, 1 (1987)

[5] A. Nitzan, Chemical Dynamics in Condensed Phases: Relaxation, Transfer, and Reactions in Condensed Molecular Systems, Oxford Graduate Texts (OUP Oxford, 2013).

[6] G. S. Agarwal, Quantum Optics (Cambridge University Press, Cambridge, 2013).

[7] M. Munowitz, Coherence and NMR (Wiley, 1988).

[8] P. Hänggi, P. Talkner, and M. Borkovec, Rev. Mod. Phys. 62, 251 (1990); E. S. Hernández and C. O. Dorso, Phys. Rev. C 29, 1510 (1984)

[9] K. Vladár, G. T. Zimányi, and A. Zawadowski, Phys. Rev. Lett. 56, 286 (1986) A. O. Caldeira, A. H. Castro Neto, and T. Oliveira de Carvalho, Phys. Rev. B 48, 13974 (1993) F. Guinea, V. Hakim, and A. Muramatsu, Phys. Rev. B 32, 4410 (1985)

[10] A. V. Ferrer and C. M. Smith, Phys. Rev. B 76, 214303 (2007) A. Villares Ferrer, A. O. Caldeira, and C. M. Smith, Phys. Rev. B 74, 184304 (2006)

[11] S. Strogatz, Nonlinear Dynamics and Chaos: With Applications to Physics, Biology, Chemistry, and Engineering. Studies in Nonlinearity (Avalon Publishing, 2014).

[12] B. der Pol, Radio Review 1, 701 (1920); B. van der Pol Jun. D.Sc., The London, Edinburgh, and Dublin Philosophical Magazine and Journal of Science 43, 700 (1922)
[13] J. Strutt (Lord Rayleigh), The theory of sound, The theory of sound No. v. 1 (Macmillan and Company, 1877).

[14] N. Levinson and O. K. Smith, Duke Math. J. 9, 382 (1942)

[15] S. Saha, G. Gangopadhyay, and D. S. Ray, International Journal of Applied and Computational Mathematics 5, $46(2019)$

[16] S. Saha, G. Gangopadhyay, and D. Shankar Ray, Communications in Nonlinear Science and Numerical Simulation 85, 105234 (2020).

[17] L. D. Landau and E. M. Lifshitz, Statistical Physics Part 1, 3rd ed. (Pergamon Press Ltd., Oxford, 1980); H. B. Callen, Thermodynamics and an Introduction to Thermostatistics, 2nd ed. (John Wiley \& Sons, Inc., New York, 1985).

[18] E. T. Owen, J. Jin, D. Rossini, R. Fazio, and M. J. Hartmann, New Journal of Physics 20, 045004 (2018).

[19] C. Navarrete-Benlloch, T. Weiss, S. Walter, and G. J. de Valcárcel, Phys. Rev. Lett. 119, 133601 (2017).

[20] M. Hillery, R. O'Connell, M. Scully, and E. Wigner, Physics Reports 106, 121 (1984)

[21] D. Barik, D. Banerjee, and D. Ray, Quantum Brownian Motion in C-numbers: Theory and Applications (Nova Science Publishers, Incorporated, 2005); A. Ghosh, S. S. Sinha, and D. S. Ray, Phys. Rev. E 86, 011122 (2012).

[22] D. Banerjee, B. C. Bag, S. K. Banik, and D. S. Ray, Phys. Rev. E 65, 021109 (2002); S. S. Sinha, A. Ghosh, and D. S. Ray, Phys. Rev. E 84, 031118 (2011), Phys. Rev. E 84, 041113 (2011).

[23] A. Ghosh, S. S. Sinha, and D. S. Ray, Phys. Rev. E 83, 061154 (2011) S. S. Sinha, A. Ghosh, B. C. Bag, and D. S. Ray, "Quantum brownian motion in a spin bath," in Concepts and Methods in Modern Theoretical Chemistry, Volume 1, edited by S. K. Ghosh and P. K. Chattaraj (Taylor \& Francis, Cham, 2013) pp. 183-204.

[24] A. Goldbeter and M. Berridge, Biochemical Oscillations and Cellular Rhythms: The Molecular Bases of Periodic and Chaotic Behaviour (Cambridge University Press, 1997).

[25] S. Kar and D. S. Ray, Phys. Rev. Lett. 90, 238102 (2003). 
[26] S. Ghosh and D. S. Ray, The European Physical Journal B 87, 65 (2014); The Journal of Chemical Physics 143, 124901 (2015)

[27] S. Linge and H. Langtangen, Programming for Computations - Python: A Gentle Introduction to Numerical Simulations with Python, Texts in Computational Science and Engineering (Springer International Publishing, 2016).

[28] P. Jung and P. Hänggi, Phys. Rev. Lett. 65, 3365 (1990)

D. Barik and D. S. Ray, Journal of Statistical Physics 120, 339 (2005)

[29] A. S. Pikovsky, M. Rosenblum, and J. Kurths, Synchronization: A Universal Concept in Nonlinear Science (Cambridge University Press, New York, 2001).

[30] J. d. M. B.van der Pol, Phil. Mag. Suppl. 6, 763 (1928).

[31] R.Fitzhugh, Biophys.J 1, 445 (1961).

[32] M. E. Jewett and R. E. Kronauer, Journal of Theoretical Biology 192, 455 (1998).

[33] T. E. Lee and H. R. Sadeghpour, Phys. Rev. Lett. 111, 234101 (2013)

[34] K. Ishibashi and R. Kanamoto, Phys. Rev. E 96, 052210 (2017)
[35] S. Walter, A. Nunnenkamp, and C. Bruder, Phys. Rev. Lett. 112, $094102(2014)$

[36] T. E. Lee, C.-K. Chan, and S. Wang, Phys. Rev. E 89, $022913(2014)$

[37] D. Leibfried, R. Blatt, C. Monroe, and D. Wineland, Rev. Mod. Phys. 75, 281 (2003).

[38] H. HAdffner, C. Roos, and R. Blatt, Physics Reports 469, 155 (2008)

[39] R. Blatt and C. F. Roos, Nature Physics 8, 277 (2012).

[40] C. Monroe and J. Kim, Science 339, 1164 (2013).

[41] L. Rayleigh, Edinburgh, and Dublin Philosophical Magazine and Journal of Science 15, 229 (1883)

[42] A. Yariv, Introduction to Optical Electronics (2000).

[43] G. S. Rychkov, Differ. Uravn. 11, 390 (1975)

[44] T. R. Blows and N. G. Lloyd, Mathematical Proceedings of the Cambridge Philosophical Society 95, 359âAŞ366 (1984)

[45] H. Giacomini and S. Neukirch, Phys. Rev. E 56, 3809 (1997)

[46] J. Llibre, L. Pizarro, and E. Ponce, Phys. Rev. E 58, 5185 (1998).

[47] S. Ghosh and D. S. Ray, The Journal of Chemical Physics 139, 164112 (2013)

[48] V. Gaiko, Cubo 10, 115 (2008).

\section{APPENDIX}

\section{A. Derivation of Eq. 37}

Consider the operator Langevin equation (29) which is also the special case of generalized Langevin equation (9) for $n=1$. Taking average on both sides of the equation with the initial product separable coherent states of system and bath oscillators $|\alpha\rangle\left|\mu_{1}\right\rangle\left|\mu_{2}\right\rangle \ldots\left|\mu_{N}\right\rangle$ one finds

$$
\dot{\alpha}=-\frac{\gamma_{2}}{m_{1}}\left(m_{1}+m_{0}\right)\left\langle\alpha\left|\hat{A}^{\dagger} \hat{A}^{2}\right| \alpha\right\rangle+\frac{\gamma_{2}}{m_{1}} m_{0}\left\langle\alpha\left|\hat{A}^{\dagger} \hat{A}^{2}\right| \alpha\right\rangle+f_{2} \alpha^{*},
$$

where we have split $-\gamma_{2}\left\langle\alpha\left|\hat{A}^{\dagger} \hat{A}^{2}\right| \alpha\right\rangle$ into two terms by introducing two dimensionless numbers $m_{0}$ and $m_{1} \cdot m_{0}$ and $m_{1}$ can assume positive or negative values depending on the specificity of the case and are independent of each other. This choice is determined by the characteristic polynomial of classical nonlinear damping. Rewriting the above equation (A1) we have

$$
\begin{aligned}
\dot{\alpha} & =-\frac{\gamma_{2}}{m_{1}}\left(m_{1}+m_{0}\right)|\alpha|^{2} \alpha+\frac{\gamma_{2}}{m_{1}} m_{0}\left\langle\alpha\left|\hat{A}\left(\hat{A}^{\dagger} \hat{A}-1\right)\right| \alpha\right\rangle+f_{2} \alpha^{*} \\
& \approx-\frac{\gamma_{2}}{m_{1}}\left(m_{1}+m_{0}\right)|\alpha|^{2} \alpha+\frac{\gamma_{2}}{m_{1}} m_{0}\langle\alpha|\hat{A}| \alpha\rangle\left\langle\alpha\left|\left(\hat{A}^{\dagger} \hat{A}-1\right)\right| \alpha\right\rangle+f_{2} \alpha^{*} \\
& =-\frac{\gamma_{2}}{m_{1}}\left(m_{1}+m_{0}\right)|\alpha|^{2} \alpha+\frac{\gamma_{2}}{m_{1}} m_{0}\left(|\alpha|^{2}-1\right) \alpha+f_{2} \alpha^{*} \\
& =-\frac{\gamma_{2}}{m_{1}}\left(m_{1}|\alpha|^{2}+m_{0}\right) \alpha+f_{2} \alpha^{*} .
\end{aligned}
$$

In deriving the Eq. A2 it has been assumed $\left\langle\hat{A}\left(\hat{A}^{\dagger} \hat{A}-1\right)\right\rangle \approx\langle\hat{A}\rangle\left\langle\left(\hat{A}^{\dagger} \hat{A}-1\right)\right\rangle$, which is a valid approximation within the mean-field theory. This corresponds to neglect of higher order quantum correlation in the dynamics of nonlinear dissipation; quantum noise due to the heat-bath, however, completely unaffected. 


\section{B. Derivation of Eq. 45}

Setting $n=2$ in Eq. (9) and averaging with $|\alpha\rangle\left|\mu_{1}\right\rangle\left|\mu_{2}\right\rangle \ldots\left|\mu_{k}\right\rangle \ldots\left|\mu_{N}\right\rangle$, we write down the c-number Langevin equation within the mean-field approximation as

$$
\begin{aligned}
\dot{\alpha} & =-\frac{\gamma_{3}}{m_{2}}\left(m_{2}+m_{1}\right)\left\langle\alpha\left|\left(\hat{A}^{\dagger}\right)^{2} \hat{A}^{3}\right| \alpha\right\rangle+\frac{\gamma_{3}}{m_{2}} m_{1}\left\langle\alpha\left|\left(\hat{A}^{\dagger}\right)^{2} \hat{A}^{3}\right| \alpha\right\rangle+f_{3}\left(\alpha^{*}\right)^{2} \\
& =-\frac{\gamma_{3}}{m_{2}}\left(m_{2}+m_{1}\right)|\alpha|^{4} \alpha+\frac{\gamma_{3}}{m_{2}} m_{1}\left\langle\alpha\left|\hat{A}^{\dagger}\left(\hat{A} \hat{A}^{\dagger}-1\right) \hat{A}^{2}\right| \alpha\right\rangle+f_{3}\left(\alpha^{*}\right)^{2} \\
& \approx-\frac{\gamma_{3}}{m_{2}}\left(m_{2}+m_{1}\right)|\alpha|^{4} \alpha+\frac{\gamma_{3}}{m_{2}} m_{1}\left\langle\alpha\left|\hat{A}^{\dagger} \hat{A}\right| \alpha\right\rangle\left\langle\alpha\left|\hat{A}^{\dagger} \hat{A}^{2}\right| \alpha\right\rangle-\frac{\gamma_{3}}{m_{2}} m_{1}\left\langle\alpha\left|\hat{A}^{\dagger} \hat{A}^{2}\right| \alpha\right\rangle+f_{3}\left(\alpha^{*}\right)^{2} \\
& =-\frac{\gamma_{3}}{m_{2}}\left(m_{2}+m_{1}\right)|\alpha|^{4} \alpha+\frac{\gamma_{3}}{m_{2}} m_{1}|\alpha|^{4} \alpha-\frac{\gamma_{3}}{m_{2}}\left(m_{1}+m_{0}\right)\left\langle\alpha\left|\hat{A}^{\dagger} \hat{A}^{2}\right| \alpha\right\rangle+\frac{\gamma_{3}}{m_{2}} m_{0}\langle\alpha|\left(\hat{A} \hat{A}^{\dagger} \hat{A}-\hat{A}\right)+f_{3}\left(\alpha^{*}\right)^{2} \\
& \approx-\frac{\gamma_{3}}{m_{2}} m_{2}|\alpha|^{4} \alpha-\frac{\gamma_{3}}{m_{2}}\left(m_{1}+m_{0}\right)|\alpha|^{2} \alpha+\frac{\gamma_{3}}{m_{2}} m_{0}\langle\alpha|\hat{A}| \alpha\rangle\left\langle\alpha\left|\hat{A}^{\dagger} \hat{A}-1\right| \alpha\right\rangle+f_{3}\left(\alpha^{*}\right)^{2} \\
& =-\frac{\gamma_{3}}{m_{2}} m_{2}|\alpha|^{4} \alpha-\frac{\gamma_{3}}{m_{2}}\left(m_{1}+m_{0}\right)|\alpha|^{2} \alpha+\frac{\gamma_{3}}{m_{2}} m_{0}\left(|\alpha|^{2}-1\right) \alpha+f_{3}\left(\alpha^{*}\right)^{2} \\
& =-\frac{\gamma_{3}}{m_{2}}\left(m_{2}|\alpha|^{4}+m_{1}|\alpha|^{2}+m_{0}\right) \alpha+f_{3}\left(\alpha^{*}\right)^{2} .
\end{aligned}
$$

Here the mean-field approximation has been applied twice to obtain the Eq. B1] or Eq. 45 . $m_{2}, m_{1}$ and $m_{0}$ are the integers to be chosen as per requirement of the form of the polynomial describing nonlinear dissipation.

\section{Derivation of Eq. 47}

Following the same procedure as above, it may be anticipated that we can put $n=k$ in Eq. (9) and generate terms like $|\alpha|^{2 k} \alpha,|\alpha|^{2 k-2} \alpha \ldots$ upto $|\alpha|^{2} \alpha$ using c-number formalism. The basis of this assumption lies on the previous two cases. Therefore we can presume the structure for $n=k$ within the purview of mean-field approximation as follows

$$
\begin{aligned}
\dot{\alpha} & =-\gamma_{k+1}\left\langle\alpha\left|\left(\hat{A}^{\dagger}\right)^{k} \hat{A}^{k+1}\right| \alpha\right\rangle+f_{k+1}\left(\alpha^{*}\right)^{k} \\
& =-\frac{\gamma_{k+1}}{l_{k}}\left(l_{k}|\alpha|^{2 k}+l_{k-1}|\alpha|^{2 k-2} \ldots . .+l_{1}|\alpha|^{2}+l_{0}\right) \alpha+f_{k+1}\left(\alpha^{*}\right)^{k},
\end{aligned}
$$

where the coefficients $l_{k}, l_{k-1} \ldots, l_{1}$ and $l_{0}$ may or may not be interrelated.

Now having obtained the above equation for $n=k$, if we show that the same structure holds for $n=k+1$, we may claim that we prove our desired result Eq. (47). For that we go back to Eq. (9) and take $n=k+1$ and carry out the averaging with respect to coherent states as before

$$
\begin{aligned}
\dot{\alpha}= & -\gamma_{k+2}\left\langle\alpha\left|\left(\hat{A}^{\dagger}\right)^{k+1} \hat{A}^{k+2}\right| \alpha\right\rangle+f_{k+2}\left(\alpha^{*}\right)^{k+1} \\
= & -\frac{\gamma_{k+2}}{m_{k+1}}\left(m_{k+1}-m\right)\left\langle\alpha\left|\left(\hat{A}^{\dagger}\right)^{k+1} \hat{A}^{k+2}\right| \alpha\right\rangle-\frac{\gamma_{k+2}}{m_{k+1}} m\left\langle\alpha\left|\left(\hat{A}^{\dagger}\right)^{k}\left(\hat{A} \hat{A}^{\dagger}-1\right) \hat{A}^{k+1}\right| \alpha\right\rangle+f_{k+2}\left(\alpha^{*}\right)^{k+1} \\
\approx & -\frac{\gamma_{k+2}}{m_{k+1}}\left(m_{k+1}-m\right)|\alpha|^{2 k+2} \alpha-\frac{\gamma_{k+2}}{m_{k+1}} m\left\langle\alpha\left|\left(\hat{A}^{\dagger}\right)^{k} \hat{A}\right| \alpha\right\rangle\left\langle\alpha\left|\hat{A}^{\dagger} \hat{A}^{k+1}\right| \alpha\right\rangle \\
& +\frac{\gamma_{k+2}}{m_{k+1}} m\left\langle\alpha\left|\left(\hat{A}^{\dagger}\right)^{k} \hat{A}^{k+1}\right| \alpha\right\rangle+f_{k+2}\left(\alpha^{*}\right)^{k+1} \\
= & -\frac{\gamma_{k+2}}{m_{k+1}}\left(m_{k+1}-m\right)|\alpha|^{2 k+2} \alpha-\frac{\gamma_{k+2}}{m_{k+1}} m|\alpha|^{2 k+2} \alpha \\
& +\frac{\gamma_{k+2}}{m_{k+1}} \frac{m_{k}}{l_{k}}\left\{l_{k}|\alpha|^{2 k}+l_{k-1}|\alpha|^{2 k-2} \ldots+l_{1}|\alpha|^{2}+l_{0}\right\} \alpha+f_{k+2}\left(\alpha^{*}\right)^{k+1} \\
= & -\frac{\gamma_{k+2}}{m_{k+1}}\left(m_{k+1}-m\right)|\alpha|^{2 k+2} \alpha-\frac{\gamma_{k+2}}{m_{k+1}} m|\alpha|^{2 k+2} \alpha \\
& -\frac{\gamma_{k+2}}{m_{k+1}}\left(m_{k}|\alpha|^{2 k}+m_{k-1}|\alpha|^{2 k-2} \ldots+m_{1}|\alpha|^{2}+m_{0}\right) \alpha+f_{k+2}\left(\alpha^{*}\right)^{k+1} \\
= & -\frac{\gamma_{k+2}}{m_{k+1}}\left(m_{k+1}|\alpha|^{2 k+2}+m_{k}|\alpha|^{2 k} \ldots+m_{1}|\alpha|+m_{0}\right) \alpha+f_{k+2}\left(\alpha^{*}\right)^{k+1}
\end{aligned}
$$

Here we use Eq. (C1) in Eq. C2). Further defining

$$
m_{j}=-\frac{l_{j}}{l_{k}} m, \quad \forall \quad j=0,1,2 \ldots k .
$$


in Eq. (C3) we obtain Eq. (C4). Thus we find the same structure for $n=k+1$ as for $n=k$. Therefore by the method of mathematical induction we have proved that the structure assumed in Eq. (47) is true for all $n \in \mathbb{Z}^{+}$. Similar technique can be followed to derive its complex conjugate equation.

\section{Rayleigh Family of Quantum Oscillators}

From c-number Langevin Eqs.47) and (48) we construct the following form of the quantum equation with the help of substitution 42

$$
\ddot{x}+\frac{\gamma_{n+1}}{m_{n}}\left\{\beta_{n} \dot{x}^{2 n}+\beta_{n-1} \dot{x}^{2 n-2} \ldots . .+\beta_{1} \dot{x}^{2}+\beta_{0}\right\} \dot{x}+\omega_{0}^{2} x=\omega_{0}^{n-1} \eta_{n+1} .
$$

This Eq. (D1) can be considered as quantum analog of generalized Rayleigh family of oscillators [13, 47, where

$$
\beta_{j}=\frac{m_{j} j}{{ }^{2 j} C_{j+1}(2 j+1)}, \quad \forall j=1,2, \ldots . n \quad \text { and } \quad \beta_{0}=m_{0} .
$$

The family of oscillators could also support at most $n$ number of limit cycles, where $2 n$ is the highest degree of the polynomial [48. The phase space trajectories of a representative example of such generalized Rayleigh oscillator is shown in both (a) classical and (b) quantum picture in Fig. (5).
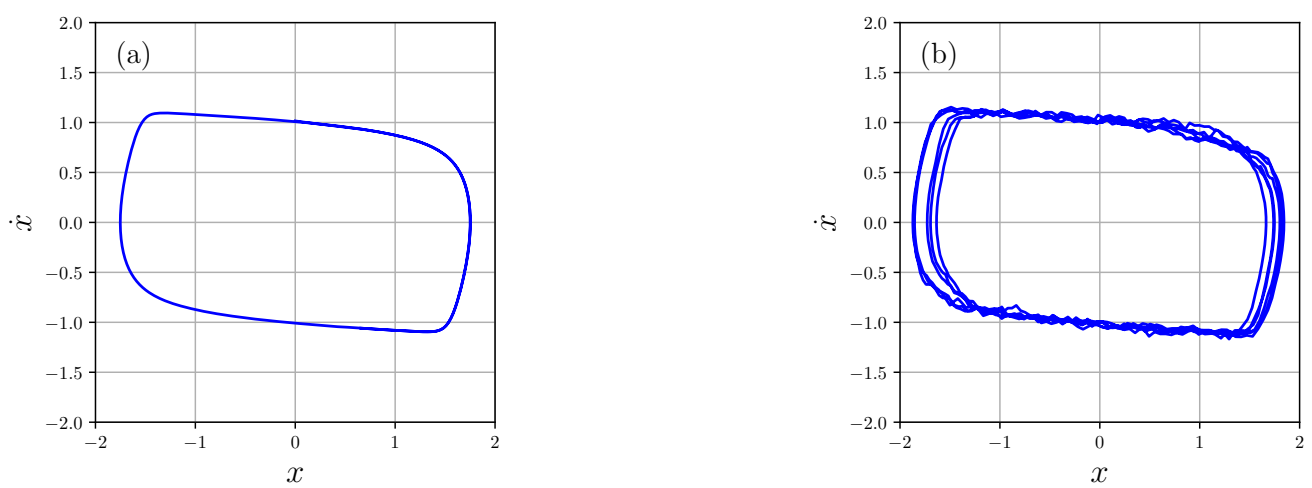

FIG. 5. The phase space trajectories of (a) classical and (b) quantum version of a generalized Rayleigh oscillator, for polynomial $\dot{x}^{6}+\dot{x}^{4}-\dot{x}^{2}-1$ are plotted for $\epsilon=1.5$. We have put $K T=20$ for plotting the quantum case (units arbitrary). 